\title{
The use of Kirlian photography in preventive medicine and the education
}

\author{
L.A. Pesotskaya ${ }^{1}$, T.V. Lakiza ${ }^{1}$, N.V. Glukhova ${ }^{2}$, T.O. Tretiak ${ }^{3}$ \\ ${ }^{1}$ Dnipropetrovsk medical Academy of Ministry of health of Ukraine \\ ${ }^{2}$ National mining University, Dnipro, Ukraine \\ ${ }^{3}$ National University name Oles Honchar \\ *e-mail:lpesotskaya23@gmail.com
}

Key words: Kirlian photography, preventive medicine, REK-1

The aim of our work was to study a state of health at children and adults using a method of Kirlian photography before and after influence of improving actions homoeopathic, fito - preparations, minerals.

For this inspections applied device "REK-1", developed in Dnepropetrovsk. Researches were held on a X-ray film. It's analysis were held on by diagnostic criteria P. Mandel and results of our observations. A normal luminescence crown around fingers is represented by an inner oval, a middle luminescence layer in the form of evenly located streamers. Inspected the 86 children at the age from 8 till 15 years from families of liquidators of consequences of Chernobyl. The comparative analysis of Kirlian-photos after stay in sanatorium has revealed positive dynamics in an energy- information's condition of an organism at the majority of children. The best results in the threat and endocrinr zones are received in group of children receiving homoeopathic preparations.

Kirlian-diagnostics have been tried on 57 workers of one of the industrial enterprises. As a Kirlian-luminescence at everyone level reactions of adaptation has been established and therapy by phytopreparations

The Kirlian-diagnostic was on 40 persons before stay during 10-20 minutes in clay or shungite rooms. At the moment of inspection people were almost healthy or had diseases out of an aggravation. The phenomena of emotional liability, dysfunction endocrine regulation equally effectively decreased in both groups.

Kirlian-diagnostic's method examined 56 students of Junior and senior courses of study at the University. At pupils on the basis of psychological tests types of thinking were defined: figurative, logical, intuitive. The obtained results were compared with the results of the analysis area of the crown's fluorescence of the fingers in the Kirlian photos after each test. Revealed differences in Kirlian images with different types of thinking. Activation of different reactive systems of the organism was observed in according to the age.

The Kirlianography method of human adaptation reserves functional state research should be used in health-improving and medical practice, ecological population healthimproving programmes for the estimation of the negative factors of the environment or the existing disease as well as the choice of a needed therapy.

It is advisable to use kirlianography in the pedagogical process to adapt it to the prevailing type of thinking in the group of students. 